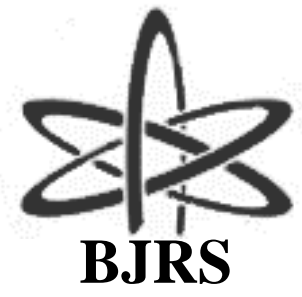

\author{
BRAZILIAN JOURNAL \\ $\mathrm{OF}$ \\ RADIATION SCIENCES \\ 08-02 (2020) 01-09
}

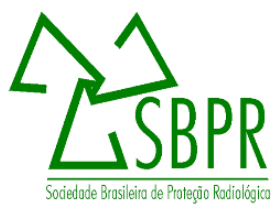

\title{
Relative depth-doses from square and rectangular Y-90 beta applicators used in skin cancer therapy
}

\author{
Eduardo De Paiva \\ Division of Medical Physics, Institute of Radiation Protection and Dosimetry - IRD/CNEN \\ 22783-127, Rio de Janeiro, Brazil \\ edup2112@gmail.com
}

\begin{abstract}
Plaques containing beta-emitting isotopes have several medical applications. In particular, due to the short range of beta particles and the rapid fall off of dose rates they are suitable to be used in small and superficial skin cancer therapy, killing the tumor cells and preserving the underlying healthy structures. The analytical/numerical beta-point dose function formalism is used to a simple estimate of the relative dose rates around square and rectangular beta applicator containing the Yttrium-90 isotope. Maximum differences in relative dose rates along central axis of $13 \%$ at $6.6 \mathrm{~mm}$ depth and $20 \%$ at $5 \mathrm{~mm}$ depth were found, respectively, for the square and rectangular plaques as compared to the literature measurements data. Results have also indicated that for both plaques dose rates fall off to $50 \%$ of the reference value at about $2 \mathrm{~mm}$ depth; drop to just $10 \%$ at $4.5 \mathrm{~mm}$ depth and become zero from $8 \mathrm{~mm}$ on. This fact confirms that these sources are suitable to be used in superficial skin cancer therapy. The method presented here may be used to estimate the relative dose rates around square and rectangular Y-90 beta applicators in a simple and fast way.
\end{abstract}

Keywords: Skin cancer therapy, beta particles, Y-90 applicator, beta-point dose function, relative depth-dose calculation. 


\section{INTRODUCTION}

Beta radiation may be used in various medical applications [1]. Planar as well concave sources containing the beta emitting nuclides ${ }^{90} \mathrm{Sr} /{ }^{90} \mathrm{Y}$ can be used for the post-operative treatment of pterygia, and for the treatment of keloid and glioblastoma; small seeds of ${ }^{90} \mathrm{Sr} /{ }^{90} \mathrm{Y}$ can also be used in intravascular brachytherapy to prevent the re-closing of arteries after balloon angioplasty. Concave applicators containing ${ }^{106} \mathrm{Ru} /{ }^{106} \mathrm{Rh}$ can be used in ophthalmic brachytherapy for the treatment of choroid melanomas and retinoblastomas; beta emitting wire sources of ${ }^{32} \mathrm{P}$ can be also used in intravascular brachytherapy to prevent restenosis; recently, a feasibility study of the use of a circular patch containing microspheres of Y-90 to treat small skin lesions and estimates of relative dose rates to this applicator were published [2,3].

Beta particles have a short range within the tissue and a high-dose gradient. These properties play an important role in the treatment of small and superficial skin lesions, provided that the beta sources deliver a high dose in a few millimeters thus preserving the neighboring healthy tissues and personnel, making the radiation protection a simple task. Skin cancer is the most common kind of malignancy all over the world and the use of beta radiation to treat small skin lesions is particularly interesting in that cases where surgery is not possible or recommended [4].

However, a direct and accurate dosimetry of dose distributions around brachytherapy applicators containing pure beta emitters is very difficult to carry out, mainly due to the short range of the betaparticles with respect to the size of detectors, the steep high-dose gradients, a non-uniform distribution of the radionuclide over the plaque surface or volume, and in some cases the asymmetric geometry of the plaques. As a consequence, there are a few experimental data on these plaques and generally with large discrepancies among them. Note for example that, for commercially available ${ }^{106} \mathrm{Ru} /{ }^{106} \mathrm{Rh}$ curved plaques (produced by Eckert \& Ziegler BEBIG, GmbH, Berlin, Germany) used in ophthalmic brachytherapy, the dose rates along central axis are provided with an uncertainty of $\pm 20 \%$. Thus, calculation methods are of great importance. There are two approaches to estimate dose rates around beta emitting applicators. Monte Carlo (MC) simulations, that makes possible to manage with complex geometries and various kind of media to obtain results with great accuracy. Nevertheless, the use of MC simulations may require a large time of programming and computation and may not be user friendly in a daily clinical routine. Another way to estimate the dose rates is by means of 
analytical/numerical methods, that apply only to homogeneous medium (water) and to simple geometries, but has the advantage of requiring a negligible time of computation.

In this work we present a simple analytical/numerical method to estimate the relative dose rates along central and lateral axes of square and rectangular plaques containing the beta-emitting Yttrium90 isotope intended to be used in superficial skin cancer treatment and a comparison is made with available experimental data obtained using radiograph film and Fricke xylenol gel dosimeter (FXG) [5]. In the results presented in this study it was considered radioactive plaques with the same properties and dimensions as those reported in the experimental study [5].

\section{MATERIALS AND METHODS}

The dose rate around an extended beta-emitting source can be calculated using the formalism introduced by Loevinger [6]. Loevinger presented an empirical expression based on measurements of beta transmission through sheets of polystyrene and it is known as the beta-point source dose function.

In Figure 1 is depicted the geometry used through calculations. The absorbed dose rate $J(\xi)$, the beta-point source dose function, due to a beta particle located in point $\mathrm{P}_{1}(x, y, z)$ on the plaque at a point $\mathrm{P}_{0}\left(x_{0}, y_{0}, z_{0}\right)$ within the tissue is given by [6,7]

$$
J(\xi)=\frac{B}{(\rho \nu \xi)^{2}}\left\{c\left[1-\frac{\rho \nu \xi}{c} \exp \left(1-\frac{\rho \nu \xi}{c}\right)\right]+\rho v \xi \exp (1-\rho v \xi)-\rho v \xi \exp \left(1-\frac{\rho v \xi}{2}-\frac{f}{2}\right)\right\}
$$

where $\xi$ is the distance from $\mathrm{P}_{1}$ to $\mathrm{P}_{0}$ as shown in Figure 1,

$$
\xi=\sqrt{\left(x-x_{0}\right)^{2}+\left(y-y_{0}\right)^{2}+\left(z-z_{0}\right)^{2}} .
$$

$\rho$ is the density of the homogeneous medium (water), $v$ is the absorption coefficient, and $c$ and $f$ are dimensionless parameters that satisfy 


$$
\left[1-\frac{\rho v \xi}{c} \exp \left(1-\frac{\rho v \xi}{c}\right)\right] \equiv 0 \quad \text { for } \rho v \xi \geq c
$$

and

$$
J(\xi) \equiv 0 \text { for } \rho v \xi \geq f
$$

The factor $B$ represents a normalization constant given by $B=0.046 \rho^{2} v^{3} E_{\beta} \alpha$, where $E_{\beta}$ is the mean kinetic energy of the beta particles, and $\alpha$ is related to parameters $c$ and $f$ by means of

$$
\frac{1}{\alpha}=3 c^{2}-\left(c^{2}-1\right) \exp (1)+(3+f) \exp (1-f)-4 \exp \left(1-\frac{f}{2}\right)
$$

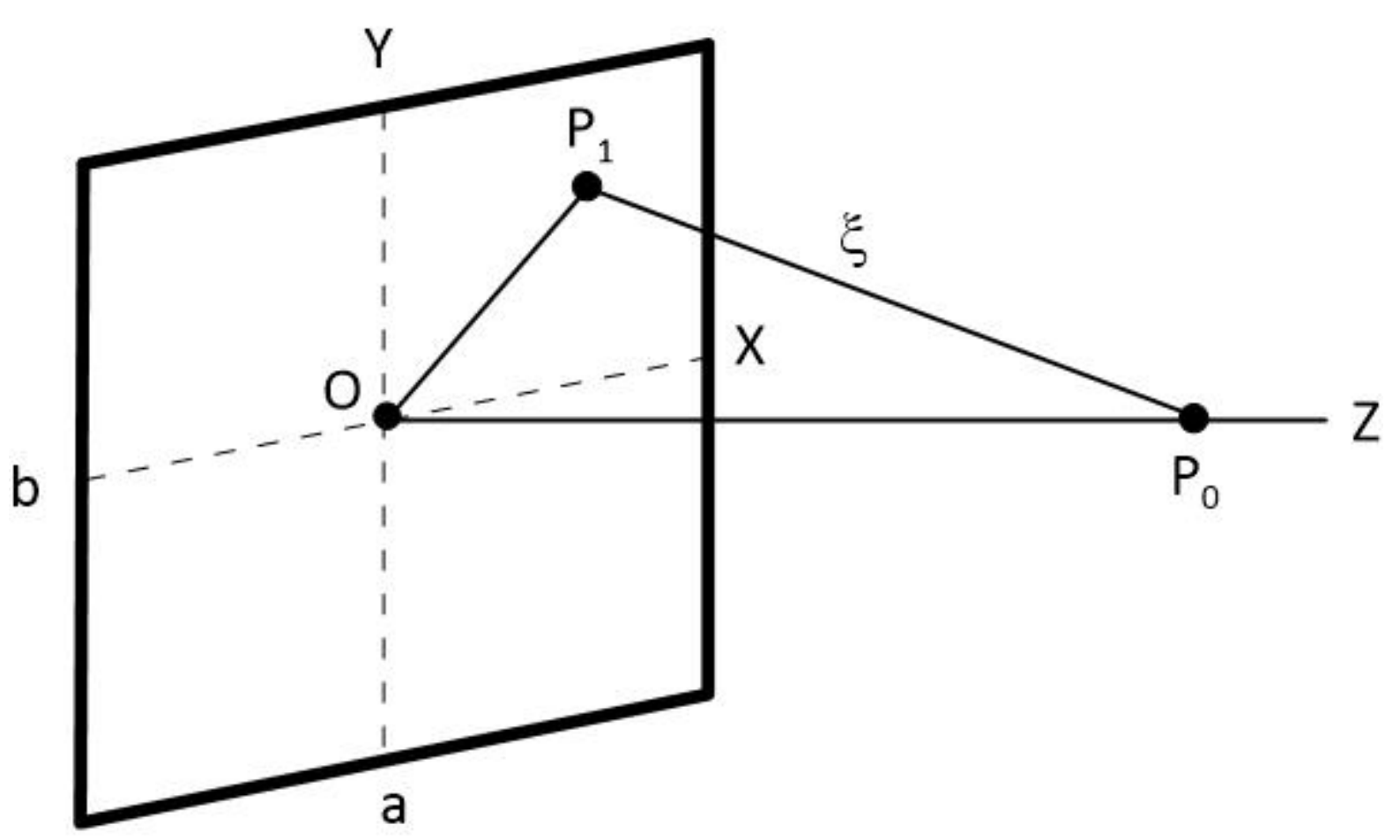

Figure 1: The geometry of the square and rectangular Y-90 plaques used in calculations 
The first term in Equation (1) accounts for the energy absorbed from the un-scattered component of the beta particles and is zero at distances $\xi \geq c / \rho v$. Equation (1) is a modification presented by Vynckier and Wambersie [7] of the initial expression proposed by Loevinger [6] in order to incorporate new experimental and theoretical data. For this reason, the parameter $f$ was introduced and $f / \rho v$ is the distance from which the dose due to beta-particles is zero.

The absorbed dose rate $\dot{D}$ at point $\mathrm{P}_{0}$ is obtained by summing up the contribution of all points on the plaque,

$$
\dot{D}=a_{S} \iint J(\xi) \cdot d S
$$

where $a_{S}$ is the surface activity and $d S$ is the area element. In Equation (6) we make the assumptions that the eye is formed by a water-equivalent medium; the beta-emitting material is uniformly deposited on the planar surface of the applicator, and there is no encapsulation of the radioisotope on the plaque. The limits of integral (6) are $-a / 2 \leq x \leq a / 2$ and $-b / 2 \leq y \leq b / 2$, where $a$ and $b$ define the size of the plaque.

The double integration described above was numerically evaluated for points on the central and lateral axes of the square and rectangular Y-90 sources (half-life of 64 hours, maximum beta energy of $2.27 \mathrm{MeV}$ ) by means of a Fortran code based on the trapezoidal rule, and for a sake of comparison the results are compared to available experimental data obtained with radiograph film and FXG dosimeter [5].

\section{RESULTS AND DISCUSSION}

In the above equations the density of the medium is $1 \mathrm{~g} / \mathrm{cm}^{3}$; the parameters $v, c$ and $f$ are, respectively, $5.05 \mathrm{~cm}^{2} / \mathrm{g}, 0.95$ and 4.48; the mean kinetic energy of the beta particles is $0.933 \mathrm{MeV}$. These parameters depend on the type of beta emitting radioisotope and were obtained by fitting the beta point-dose function (Eq. 1) to beta-ray point-source dose distributions data in water obtained from MC calculations as described in ref. [1]. The rectangular and square plaques have dimensions 1 
$\mathrm{cm} \times 2 \mathrm{~cm}$ and $2 \mathrm{~cm} \times 2 \mathrm{~cm}$, and the surface activity value does not affect the results of relative doses. In Figure 2 are shown the dose rates along central axis of the square (top) and rectangular (bottom) plaques normalized at the depth of reference of $1 \mathrm{~mm}$ [1]. For comparison experimental results described in literature are also shown [5].

Maximum differences from the estimates to the mean measurements values obtained using film and FXG dosimeters along central axis of $13 \%$ at $6.6 \mathrm{~mm}$ depth and $20 \%$ at $5 \mathrm{~mm}$ depth were found, respectively, for the square and rectangular plaques. In the depths of clinical interest (up to $4 \mathrm{~mm}$ ) the differences were less than $6 \%$ for the square source and less than $15 \%$ for the rectangular source. For both plaques calculations have indicated that dose rates fall off to $50 \%$ of dose rate in the reference point at $2.1 \mathrm{~mm}$ depth; fall off to just $10 \%$ at $4.5 \mathrm{~mm}$ depth and tend to zero from $8 \mathrm{~mm}$ on. It means that the beta radiation doses are mainly absorbed within the first layers of the skin tissue, as required for a superficial skin cancer therapy.

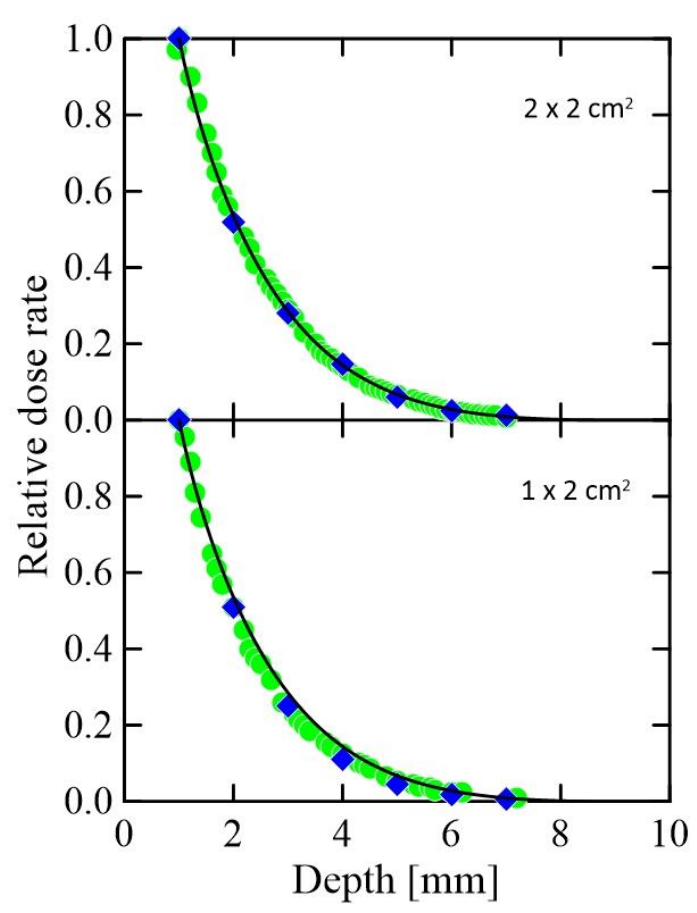

Figure 2: Depth-doses from square and rectangular plaques normalised at $1 \mathrm{~mm}$ depth. Solid lines, this work; circles, data obtained using radiograph film taken from [5]; lozenges, data obtained using FXG dosimeter taken from [5]. 
In Figures 3 and 4 the relative lateral dose rates for the two plaques are shown at the depths of 1, 2, 3, 4 and $5 \mathrm{~mm}$ and a clear effect of the size of plaques can be seen.

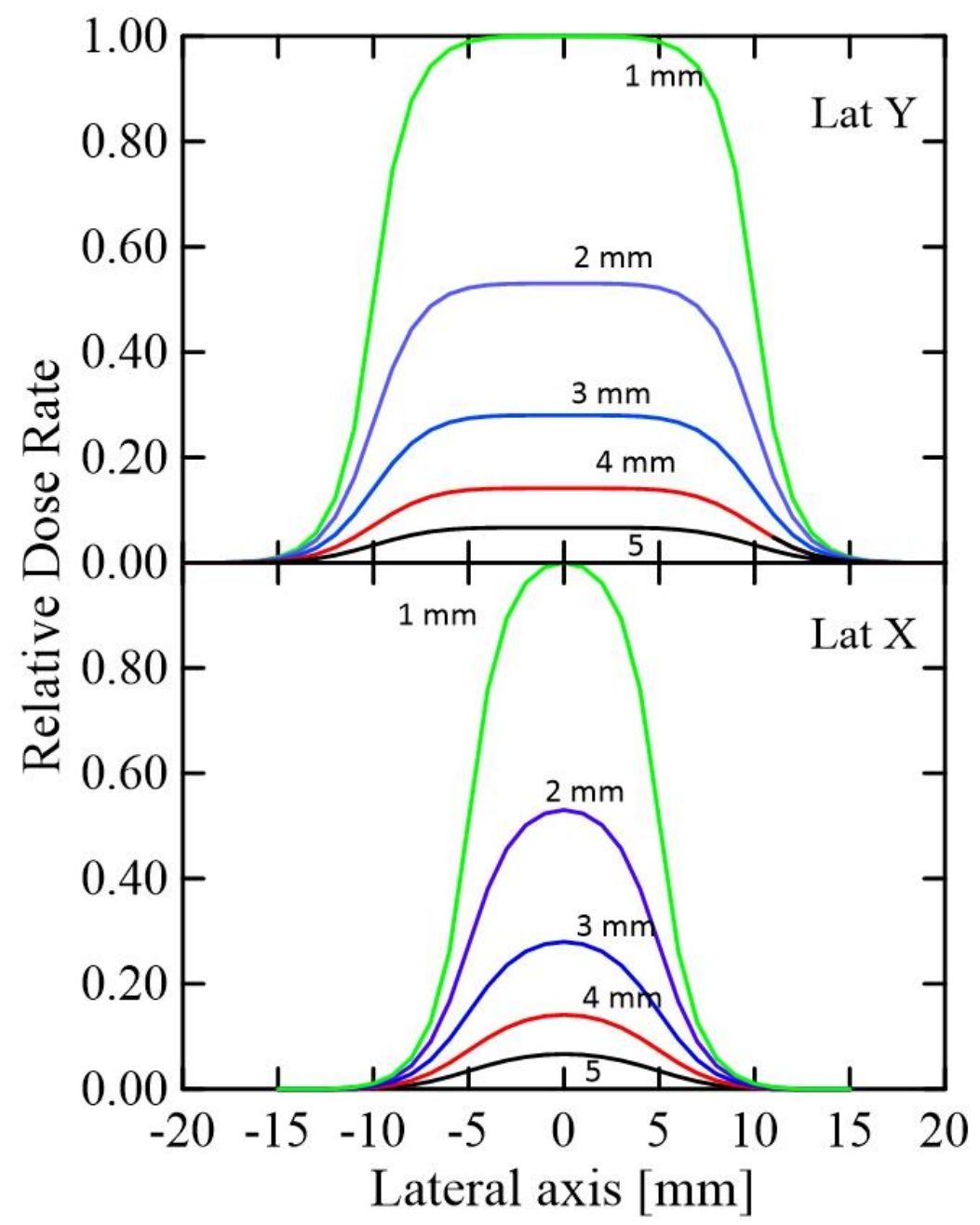

Figure 3: Relative lateral dose rates for the rectangular plaque at 1, 2, 3, 4 and $5 \mathrm{~mm}$ depth. Top, major side along y direction; bottom, minor side along $x$ direction. 


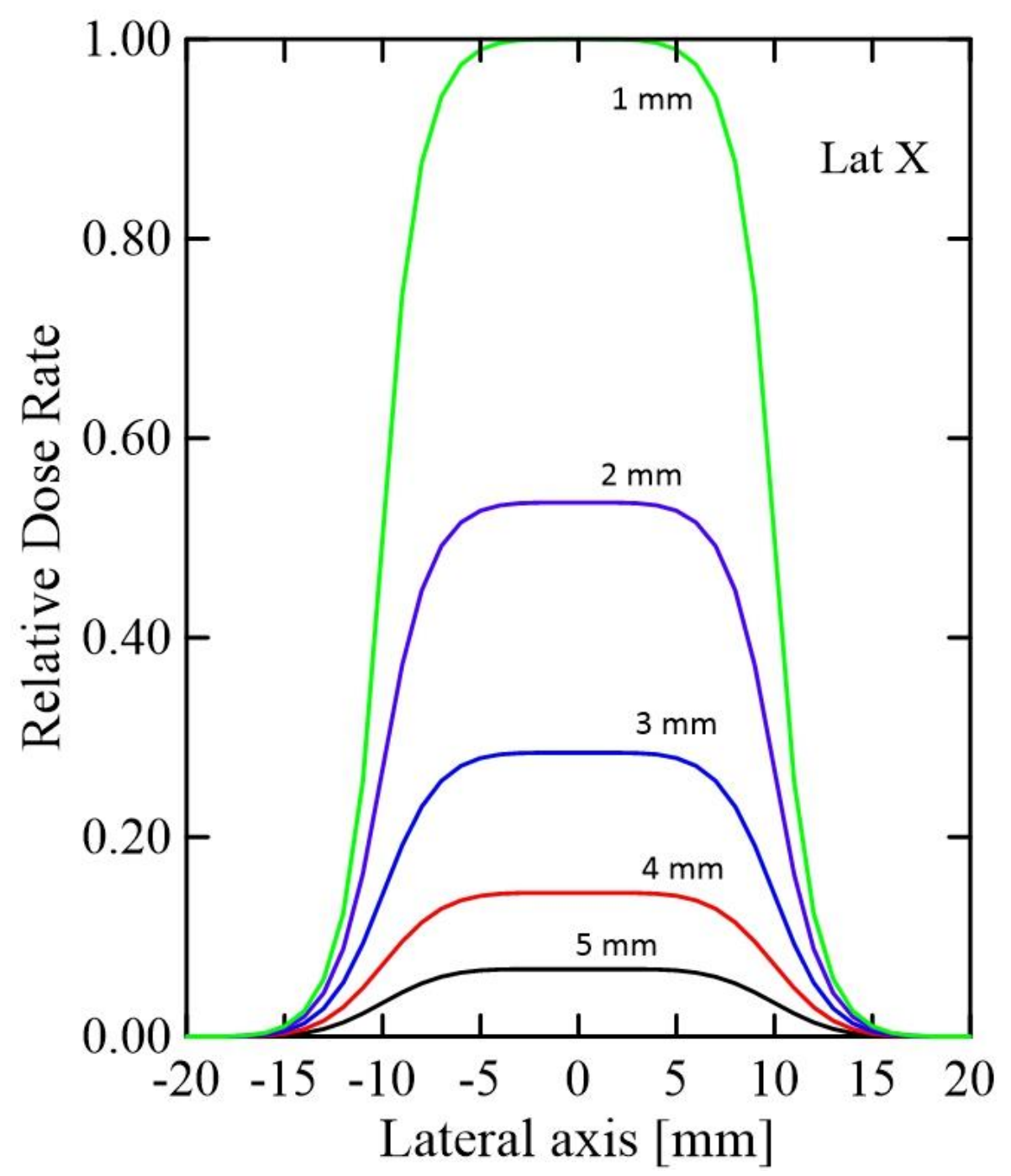

Figure 4: Relative lateral dose rates for the square plaque at 1, 2, 3, 4 and $5 \mathrm{~mm}$ depth.

\section{CONCLUSIONS}

In this work a simple estimate of the relative dose rates around a square and rectangular Y-90 beta applicators intended to be used in superficial skin cancer treatment is presented. Calculations are based on the beta-point source dose function and a reasonable agreement was found with available measurements data. Despite the limitations of the approach used the method was able to reproduce the general trend of the depth-doses around the planar plaques and results may serve as a guide to the 
next studies on them. Furthermore, we consider that the developing of a calculation routine based on a Fortran language code is the main contribution of this work since the calculation routine can be easily adapted to handle with square and rectangular plaques of various dimensions and formed by different radionuclides to estimate relative dose rates alongside central axis.

\section{REFERENCES}

[1] INTERNATIONAL COMMISSION ON RADIATION UNITS AND MEASUREMENTS. Dosimetry of beta rays and low-energy photons for brachytherapy with sealed sources. ICRU Report 72, Bethesda: ICRU, 2004.

[2] PASHAZADEH, A. et al. Superficial skin cancer therapy with Y-90 microspheres: A feasibility study on patch preparation. Skin Res Technol, v. 26, p. 25-29, 2020.

[3] PASHAZADEH, A.; DE PAIVA, E.; MAHMOODIAN, N.; FRIEBE, M. Calculation of beta radiation dose of a circular Y-90 skin patch: Analytical and simulation methods. Rad Phys Chem, v. 166, p. 108491, 2020.

[4] PASHAZADEH, A.; BOESE, A.; FRIEBE, M. Radiation therapy techniques in the treatment of skin cancer: an overview of the current status and outlook. J Dermatolog Treat, v. 30, 1-41, 2019.

[5] ALVA-SÁNCHES, M.S. et al. Beta planar source quality assurance with the Fricke xylenol gel dosimeter. Rad Phys Chem, v. 96, p. 56-59, 2014.

[6] LOEVINGER, R. The Dosimetry of Beta Sources in Tissue. The Point-Source Function. Radiol, v. 66, p. 55-62, 1956.

[7] VYNCKIER, S.; WAMBERSIE, A. Dosimetry of beta sources in radiotherapy I. The beta point source dose function. Phys Med Biol, v. 27, p. 1339-1347, 1982. 